\title{
Estratégia\&Negócios
}

ISSN 1984-3372

http://www.portaldeperiodicos.unisul.br/index.php/EeN/

\section{GESTÃO DO PROCESSO DECISÓRIO: MAPEAMENTO AO TEMA CONFORME AS DELIMITAÇÕES POSTAS PELOS PESQUISADORES}

\section{DECISIONAL PROCESS MANAGEMENT: KNOWLEDGE CONSTRUCTION ABOUT THE FIELD}

\section{Leonardo Corrêa Chaves}

Universidade Federal de Santa Catarina - UFSC.

E-mail: leonardomg@gmail.com

\section{Leonardo Ensslin}

Professor Titular da Universidade Federal de Santa Catarina- UFSC.

E-mail: leonardoensslin@gmail.com

\section{Sandra Rolim Ensslin}

Coordenadora do Programa de Pós-Graduação em Contabilidade (PPGC/UFSC) e professora adjunta da Universidade Federal de Santa Catarina - UFSC.

E-mail: sensslin@gmail.com

\section{Sergio Murilo Petri}

Universidade Federal de Santa Catarina

E-mail: smpetri@gmail.com

\section{Fabrícia Silva Da Rosa}

Universidade Federal de Santa Catarina

E-mail: fabriciasrosa@hotmail.com

Recebido em 05/06/2012. Aprovado em 14/12/2012. Disponibilizado em 04/03/2013.

Avaliado pelo Sistema double blind review

R. eletr. estrat. neg., Florianópolis, v.5, n.3, p.3-27, set./dez. 2012

http://portaldeperiodicos.unisul.br/index.php/EeN/index
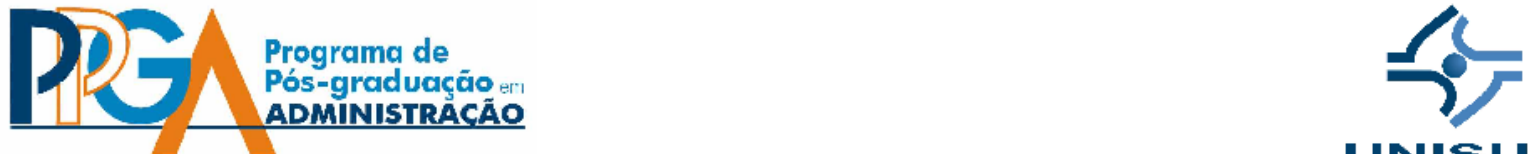

UNISUL

CCopyright 2008 UNISUL-PPGA/Estratégia e Negócios. Todos os direitos reservados. Permitida citação parcial, desde que identificada a fonte. Proibida a reprodução total. Em caso de dúvidas, consulte o editor:

ademar.unisul@gmail.com; (48) 3229-1932. 


\section{RESUMO}

A gestão do processo decisório é um assunto multidisciplinar e sua abrangência tem chamado a atenção de pesquisadores de todas as áreas, tornando interessante investigar como esse tema é tratado no meio acadêmico. Nesse sentido, o objetivo da presente pesquisa, de natureza exploratório-descritiva, consiste no mapeamento para o tema gestão do processo decisório, segundo as delimitações postas pelos pesquisadores. Para cumprir tal propósito, utilizou-se o processo Knowledge Development Process - Constructivist (ProKnow-C). Ao final do levantamento, identificaram-se 12 artigos publicados em periódicos internacionais alinhados às delimitações postas pelos pesquisadores. Na montagem do Portfólio Bibliográfico (PB), evidenciaram-se: (i) periódicos, em termos de fatores de impacto; (ii) artigos, com relação ao número de citações; (iii) os autores mais prolíferos; e, (iv) a distribuição do PB em relação aos fatores de impacto Journal Report Citattions - JCR e SCImago Journal Rank - SJR.

Palavras-chave: Processo decisório. Avaliação de desempenho. Análise bibliométrica. Gestão.

\section{INTRODUÇÃO}

A gestão do processo decisório é um assunto presente em todas as áreas do conhecimento e, devido a sua abrangência, traz importância e notoriedade, despertando forte interesse de acadêmicos e praticantes (ORDOOBADI, 2009; SALAS et al, 2010; KATSIKOPOULOS, 2011).

Com o intuito de propiciar aos atores envolvidos as condições mais favoráveis para a evolução do próprio processo e sua coerência com os valores e preferências do decisor, a gestão do processo decisório pode ser entendida como a atividade desenvolvida para auxiliar na obtenção de elementos que ajudam a compreender melhor o contexto que está sendo avaliado (ROY, 1994).

Nesse sentido, o processo decisório proporciona ao decisor um melhor entendimento do contexto a ser avaliado, permitindo-lhe um juízo de valores organizado para mensurar aquilo que considera importante e, finalmente, poder tomar decisões. Sendo assim, a decisão se restringe a ser uma parte do processo decisório. 
Foi da importância do assunto e de sua multidisciplinaridade que despertou a motivação nos pesquisadores para ampliar seu entendimento sobre o tema, fazendo surgir a seguinte indagação da pesquisa: "Como realizar um mapeamento sobre o tema Gestão do Processo Decisório, conforme as delimitações postas pelos pesquisadores?"

Desse modo, estabeleceu-se como objetivo geral deste trabalho a realização de um mapeamento sobre o tema Gestão do Processo Decisório, conforme as delimitações postas pelos pesquisadores, entendendo-se que, para tanto, será necessário:

(i) realizar um levantamento dos artigos científicos publicados em periódicos internacionais a respeito do tema "Gestão do processo decisório" para compor um Portfólio Bibliográfico (PB), conforme as delimitações postas pelos pesquisadores;

(ii) efetuar uma bibliometria no PB para ressaltar os periódicos com maior fator de impacto, artigos mais citados e autores mais citados pelo PB.

Para atingir o objetivo exposto, utilizar-se-á o processo Knowledge Development Process - Constructivist (ProKnow-C) proposto por Ensslin et al. (2010a) como instrumento de intervenção.

Além desta introdução, este artigo apresenta mais 4 seções. A seção 2 abordará o enquadramento metodológico e apresentará as premissas para a pesquisa, assim como o instrumento de intervenção. Na seção 3, realizar-se-á uma revisão de literatura sobre a gestão do processo decisório. Na seção 4, serão apresentados os resultados da pesquisa, e, finalmente, na seção 5 são apresentadas as conclusões do trabalho.

\section{METODOLOGIA}

Nesta seção, serão trabalhados o enquadramento metodológico e as premissas para a pesquisa, conjuntamente com 0 instrumento de intervenção apresentado nas seções 0,0 respectivamente. 


\subsection{ENQUADRAMENTO METODOLÓGICO}

Em relação à natureza do objetivo, trata-se aqui de uma pesquisa exploratóriodescritiva. Exploratória porque possibilitou o aprofundamento de um determinado tema, objeto do estudo. Descritiva pelo fato de descrever determinadas características do processo de pesquisa (RICHARDSON, 2008).

A lógica de pesquisa é dedutiva, considerando-se o fato de que se tinha estabelecido uma preferência pela utilização do instrumento de intervenção (IUDíCIBUS, 2004). Enquanto que o artigo apresenta uma natureza teórico-ilustrativa, exemplificada pelo assunto Gestão do Processo Decisório (FERREIRA; YOSHIDA, 2004).

Em relação ao processo de pesquisa, a coleta de dados é secundária, e a abordagem do problema é quantitativa, considerando que os artigos foram extraídos diretamente das bases de dados, visando à realização de um procedimento bibliométrico (RICHARDSON, 1999).

Este trabalho apresenta o resultado de uma pesquisa aplicada em face da geração de conhecimentos sobre o tema, até então obscurecidos para os pesquisadores (LAKATOS; MARCONI, 2008). Os procedimentos técnicos da pesquisa são de uma pesquisa bibliográfica, tendo em vista que se buscou elaborar um portfólio composto por artigos científicos.

Para atender aos objetivos desta pesquisa, foi utilizado o Knowledge Development Process - Constructivist (ProKnow-C) para realizar um mapeamento sobre o tema conforme as percepções dos pesquisadores.

O enquadramento metodológico da presente pesquisa, de forma sintetizada, consta na figura 1.

Figura 1 - Enquadramento metodológico

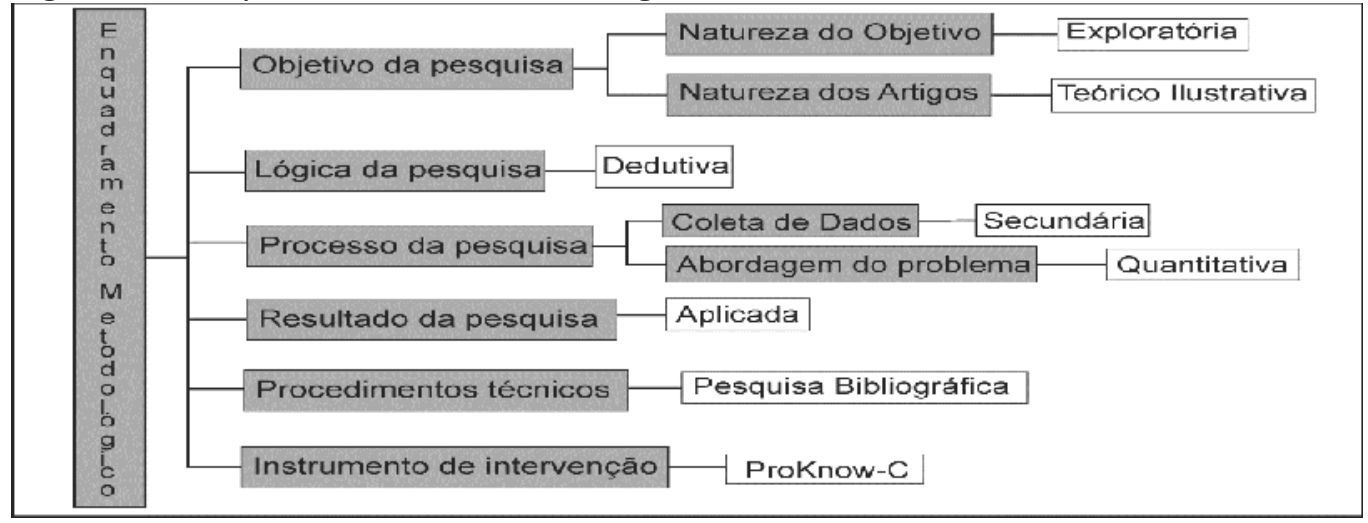

Fonte: Adaptado de Tasca et al. (2010). 


\subsection{PREMISSAS PARA A PESQUISA E INSTRUMENTO DE INTERVENÇÃO KNOWLEDGE DEVELOPMENT PROCESS - CONSTRUCTIVIST}

A ciência progride a partir da evolução do conhecimento humano, o qual é consolidado a partir de experimentos, ou da leitura de material científico. Geralmente, em um artigo científico, explicita-se qual foi o material utilizado para gerar tal conhecimento a respeito do assunto em questão. Porém, nem sempre, são explicitados os caminhos percorridos, assim como os desafios encontrados, durante a pesquisa, para se chegar a tal material.

Mesmo em artigos cuja natureza seja realizar uma revisão da literatura, o método utilizado para encontrar o material científico nem sempre é colocado de forma transparente. E essa transparência é fundamental tanto em termos metodológicos como científicos, principalmente em trabalhos que pretendam compor um arcabouço científico (HIRST et al., 2002; PATEL et al., 2004; BRANDT et al., 2005; MACLURE, 2005; LITTELL, 2008; THOMPSON et al., 2008; CALLCUT; BRANSON, 2009; LIBERATI et al., 2009; STRECH et al., 2009; BOOTH et al., 2011; IMBERGER et al., 2011; KNOBLOCH et al., 2011; MORGAN et al., 2011; ROJON et al., 2011; TRICCO et al., 2011).

Com o propósito de evidenciar os passos seguidos nesta pesquisa, deixando o leitor informado sobre os procedimentos adotados, utilizou-se como instrumento de intervenção o Knowledge Development Process - Constructivist (Proknow-C), já que este método tem como premissa explicitar as etapas da pesquisa.

O Proknow-C tem sua origem em 2009, no Laboratório Multicritério de Apoio à Decisão (LABMCDA) do departamento de Engenharia da Produção da Universidade Federal de Santa Catarina, consolidando-se a partir de 2010, quando surgiram as primeiras publicações no formato recente. O ProKnow-C é composto por 4 macroetapas, conforme se expõe na figura 2. 
Figura 2 - Macroetapas do ProKnow-C

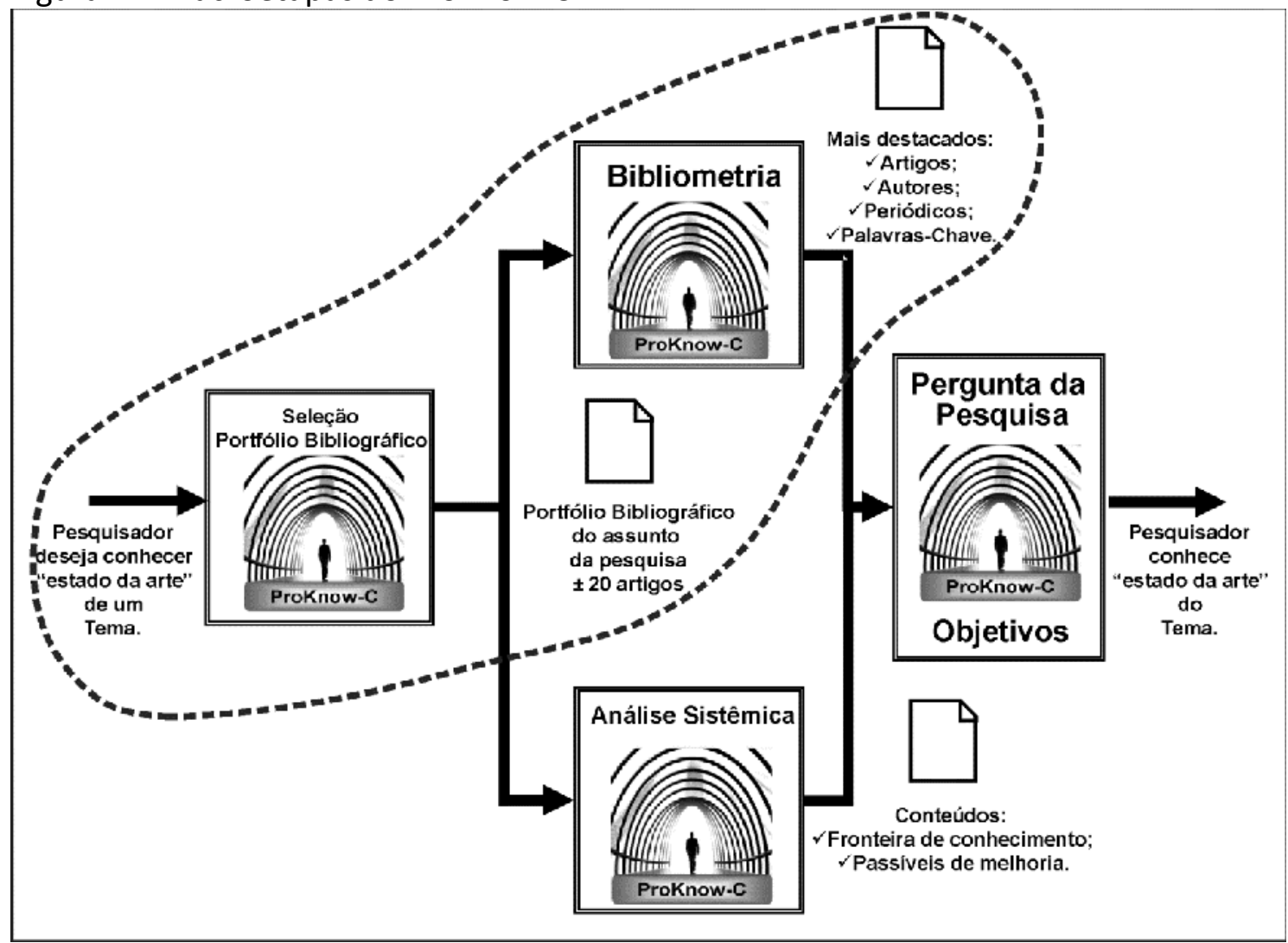

Fonte: Ensslin et al. (2012).

O propósito desse processo é propiciar melhor entendimento sobre um determinado tema a um pesquisador que deseja iniciar estudos no campo. Ao final do processo, o pesquisador terá conhecimento sobre o estado da arte. As macroetapas, tracejadas na figura 2, são as utilizadas neste trabalho.

O processo de seleção do Portfólio Bibliográfico e a Bibliometria são apresentado nas seções 0 e 0.

\subsubsection{Seleção do Portfólio Bibliográfico}

O ProKnow-C possibilita a seleção, de forma estruturada, para a construção de um Portfólio Bibliográfico (PB) de artigos com reconhecimento científico. Afonso et al. (2011, p. 51) assim se manifestam sobre o processo de seleção do Proknow-C:

A metodologia de seleção do referencial bibliográfico ProKnow-C, definida pelo LabMCDA, consiste em uma série de procedimentos sequenciais que se iniciam desde a definição 
do mecanismo de busca de artigos científicos a ser utilizado, seguindo por uma série de procedimentos pré-estabelecidos até atingir a fase de filtragem e seleção do portfólio bibliográfico relevante sobre o tema.

Os "procedimentos sequenciais", mencionados por Afonso et al. (2011), consistem na seleção de um conjunto de artigos conhecido como banco bruto de artigos e, para esse conjunto, são aplicados filtros sequenciais em relação ao alinhamento dos títulos sobre o tema, número de citações, alinhamento dos resumos e, por fim, alinhamento integral do artigo em relação ao assunto de pesquisa. O processo de seleção dos artigos do Portfólio Bibliográfico no ProKnow-C é exposto, de forma resumida, na figura 3.

Figura 3 - Processo de Seleção dos Artigos do Portfólio Bibliográfico

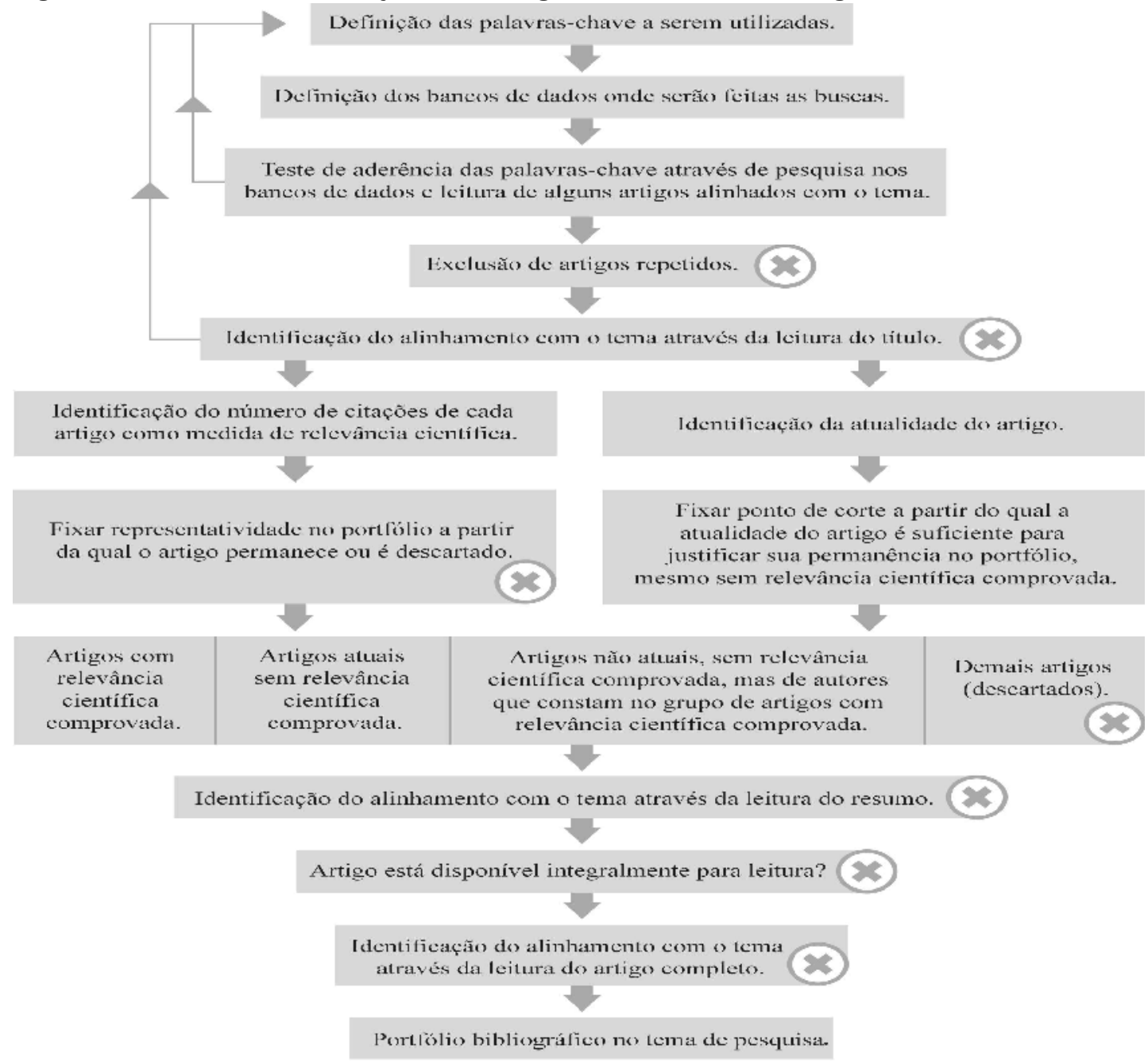

Legenda

Não obtendo bons
resultados, retornar
ao ponto inicial. $\begin{aligned} & \text { Etapa em que são } \\ & \text { descartados artigos } \\ & \text { do portfólio atual. }\end{aligned}$

Fonte: Adaptado de Afonso et al. (2011). 
Após o processo exposto na figura 3Erro! Fonte de referência não encontrada., o pesquisador tem um conjunto de material científico que representa o assunto conforme as suas próprias convicções. A partir deste material, pode-se realizar uma bibliometria sobre o tema, para conhecer quais são as variáveis de destaque.

\subsubsection{Bibliometria}

Tendo em vista o conceito de um PB, parte-se para a próxima etapa prevista pelo processo Proknow-C, a bibliometria. A bibliometria pode ser entendida como um processo para demonstrar os dados quantitativos referentes a um Portfólio Bibliográfico com o propósito de gerenciar as informações (ENSSLIN et al., 2010a).

Nesse sentido, o Proknow-C tem o propósito de realizar uma análise bibliométrica nos principais: periódicos, artigos, autores, palavras-chaves e potencial do assunto em termos de fator de impacto.

Figura 4 - Bibliometria sugerida no ProKnow-C 


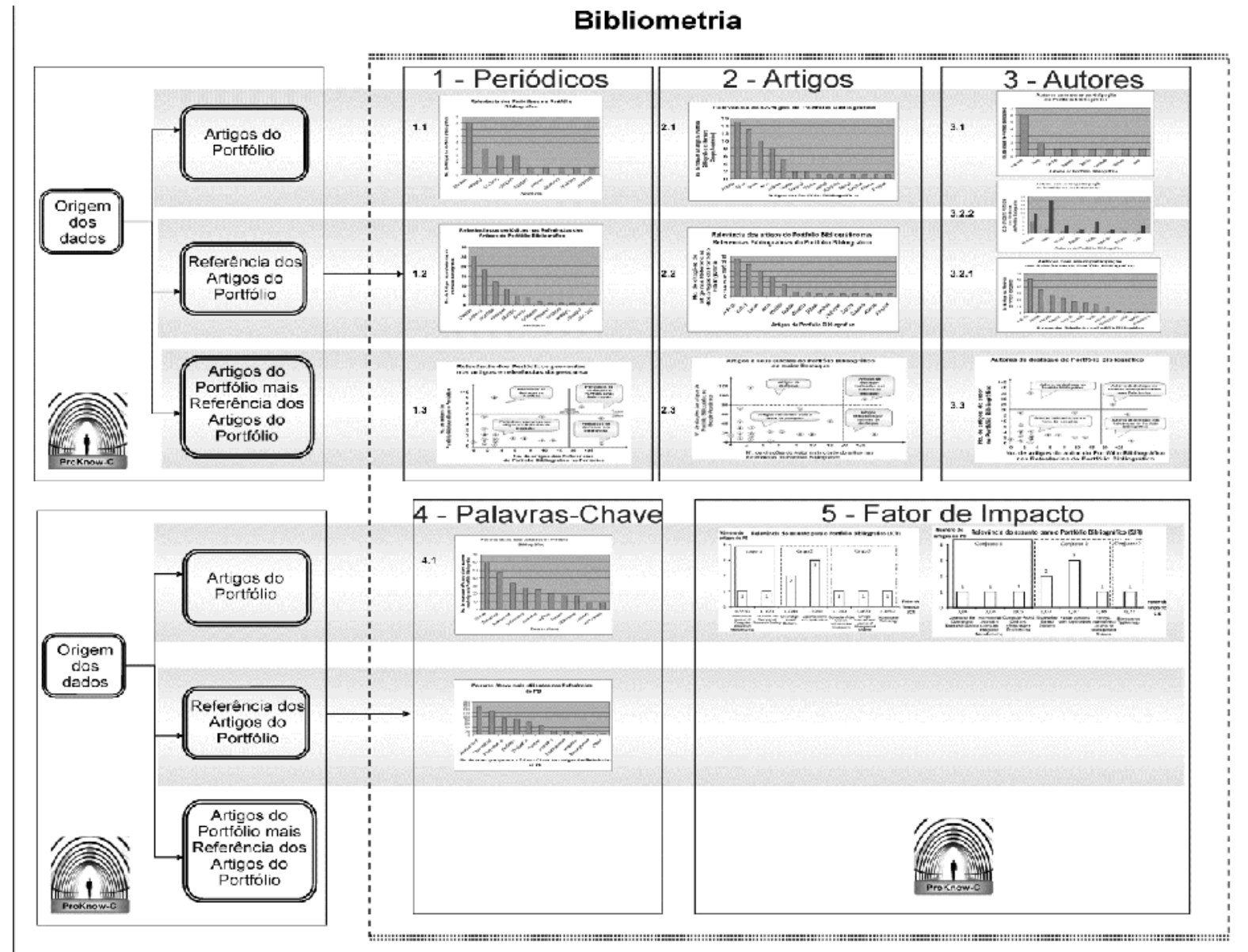

Fonte: Adaptado de Ensslin et al. (2010a).

Embora o ProKnow-C apresente alguns gráficos como padrão, diferentes gráficos sobre as mesmas variáveis (periódicos, artigos, autores, palavras-chave e fator de impacto) podem ser gerados, com o propósito de evidenciar informações sob outra ótica. Além disso, não há a obrigatoriedade de se trabalhar com todas as variáveis apresentadas no método.

O ProKnow-C hoje conta com trabalhos publicados em periódicos nacionais e internacionais. O trabalho de Bortoluzzi et al. (2011) tem como propósito construir conhecimento nos pesquisadores sobre o tema "Avaliação de Desempenho em Redes de Pequenas e Médias Empresas". Ao final, através de um portfólio composto de 41 artigos internacionais, foi verificado o European Planning Studies como periódico de maior destaque; o artigo que mais se sobressaiu foi "Small Firms, Social Capital and the Enhancement of Business Performance Through Innovation Programmes", e Ribeiro Carpinetti e Hubert Schmitz foram os autores mais prolíferos.

A pesquisa de Junior et al. (2012) tem como propósito a construção de conhecimento sobre o tema "Avaliação de desempenho na cadeia de suprimentos". Por meio de um portfólio de 
17 artigos, obteve-se como principal periódico o "International Journal of Operations \& Production Management" e, Robert Kaplan e Andy Neely como autores mais relevantes.

Outras aplicações do Proknow-C podem ser conferidas nos trabalhos de Afonso et al. (2011), Da Rosa et al. (2011), Lacerda et al. (2011), Tasca et al. (2010), Vianna et al. (2011), Lunkes et al. (2012), Valmorbida et al. (2011) e Ensslin et al. (2012).

\section{REFERENCIAL TEÓRICO - GESTÃO DO PROCESSO DECISÓRIO}

Escolha e decisão são as atividades que as pessoas realizam com maior frequência em suas vidas. Tanto na vida privada como na vida profissional, são as decisões que determinam o grau de sucesso das pessoas (STERNBERG, 2004).

O que são as decisões? São as atividades de julgamento e escolha realizadas para ter em conta aquilo que preocupa as pessoas, seus valores, objetivos, e, por fim, alcançar aquilo que desejam (LOKEN, 2007). Identificando seus objetivos, em um dado contexto, assegurará uma decisão consciente das consequências desses objetivos. São esses objetivos, valores e preocupações que a mente utiliza como pontos de referência quando fazemos julgamentos.

A habilidade para identificar esses valores, para cada contexto, é uma das principais características das pessoas bem-sucedidas, pois elas estão sempre considerando aquilo que para elas é importante. São os julgamentos de valor que permitem às pessoas expressarem suas preferências e, assim, serem coerentes em suas decisões. Decisões e julgamento estão entre os fatores que mais contribuem para as pessoas alcançarem o que desejam (STERNBERG, 2004). Até poucas décadas, o processo de julgamento se assentava em contextos estáveis, em que as mudanças podiam ser absorvidas, organizadas e tidas em conta sem a necessidade de esforços para reconhecê-las. Contudo, a crescente interdependência e complexidade da vida moderna fizeram com que, agora, o julgamento seja exercido em ambientes onde as mudanças ocorrem mais frequentemente.

Hoje, os assuntos também são de maior importância do que no passado, afetando milhares de pessoas, e a própria existência humana (HOFFMAN et al., 1998). As decisões mudaram e a forma de tomar decisões teve igualmente de mudar (MARTIN, 2002). É nesse ambiente que emerge o apoio à decisão, como instrumento para ajudar decisores a realizarem seus julgamentos, 
em uma forma mais coerente e com o conhecimento de como seus valores e preferências serão afetados. Roy (1994, p. 1) assim se manifesta sobre o que é o "Apoio à Decisão":

[...] the activity of who, in ways we call scientific, helps to obtain elements clarifying decisions in order to provide actors with the most favorable conditions possible for the type of behavior which will increase coherence between the evolution of the process, on the one hand, and the goals and/or systems values within which these actors operate on the other.

Essas condições orientam que a atividade de apoio à decisão se propõe a ser um instrumento para fornecer as condições mais favoráveis para ajudar o gestor a ser coerente com seus objetivos e preferências durante o processo decisório. O desenvolvimento do apoio à decisão necessita ter em conta o ambiente e a participação do decisor em todas as etapas.

É considerando essas condições que os pesquisadores, neste trabalho, assumem a postura de visualizar a Gestão do Processo Decisório através dos Sistemas de Apoio à Decisão, para auxiliar no próprio processo decisório, e sob a ótica da Avaliação de Desempenho para que possibilite a mensuração das consequências dos resultados (ENSSLIN et al., 2010b).

A literatura disponibiliza diversos sistemas que auxiliam a gestão do processo decisório, conhecido como Decision Support Systems. O FAHP, utilizado por Lee et al. (2008), mensura a contribuição do departamento de Tecnologia da Informação - TI nos objetivos estratégicos organizacionais. Choy et al. (2005) aplicam o Case-Based Reasoning (CBR) para seleção de fornecedores. Xirogiannis et al. (2008) utilizam o software Visual Fuzzy Cognitive Maps (Visual FCM) para saber como o departamento de RH contribui com a estratégia empresarial para satisfazer os interesses dos acionistas.

\section{RESULTADOS DA PESQUISA}

Para atingir o objetivo do presente trabalho, isto é, a construção de conhecimento nos pesquisadores sobre o tema Gestão do Processo Decisório, serão utilizadas 2 fases do processo Proknow-C. A primeira é referente à elaboração de um Portfólio Bibliográfico (PB) sobre o tema, conforme a percepção dos pesquisadores. A segunda refere-se ao processo de bibliometria, que propiciará conhecimento das variáveis de destaque do tema, em termos de periódicos, artigos e autores; e potencial do assunto, em termos de fator de impacto. 


\subsection{PROCESSO PARA SELECIONAR ARTIGOS DO PORTFÓLIO BIBLIOGRÁFICO}

Para a coleta de dados, utilizou-se também o processo Proknow-C, conforme apresentado na seção 0, destacando-se que se trata de um processo estruturado e descritivo. De uma forma geral, o processo de coleta dos dados está apresentado na figura 5, e o processo descritivo está contido no restante desta seção. A pesquisa para a seleção de Portfólio Bibliográfico foi efetuada entre março e abril de 2011.

Foram criados 2 eixos de pesquisa que representassem a percepção dos pesquisadores referente ao tema de pesquisa. Cada eixo de pesquisa tem um conjunto de palavras que o representa, quais sejam: (i) Sistemas de Apoio à Decisão e (ii) Avaliação de Desempenho.

Para o eixo Sistemas de Apoio à Decisão, foram designadas as seguintes palavraschave: Decision Support System; DSS; Technology Adoption; Information Systems; e Management System. O eixo Avaliação de Desempenho é representado pelas palavras: Performance Evaluation; Performance Assessment; Performance Appraisal e Performance Measurement. Dessa maneira, conclui-se a fase de definição das palavras-chave.

A próxima etapa consiste da definição das bases de dados a serem trabalhadas, extraídas do site da CAPES, por delimitações postas pelos pesquisadores. Após uma leitura das bases disponíveis no portal, na seção Coleções, foram identificadas 4 bases, que se encaixavam com o perfil desta pesquisa: Isiknowledge, Scopus, Willey e Ebsco.

Foram, então, pesquisados os artigos potenciais nas bases escolhidas, utilizando-se como filtro um recorte temporal de 2001 até 2011. Foram realizadas 20 buscas em cada banco de dados escolhido, a partir da combinação de cada palavra-chave do eixo Sistemas de Apoio à Decisão com as palavras-chave que representam o eixo Avaliação de Desempenho. Essas combinações de palavras foram pesquisadas nos campos: título, resumo e palavras-chave. Por fim, como último filtro, aceitaram-se somente artigos científicos publicados em periódicos para a consulta.

Figura 5 - Processo de busca dos artigos no PB 


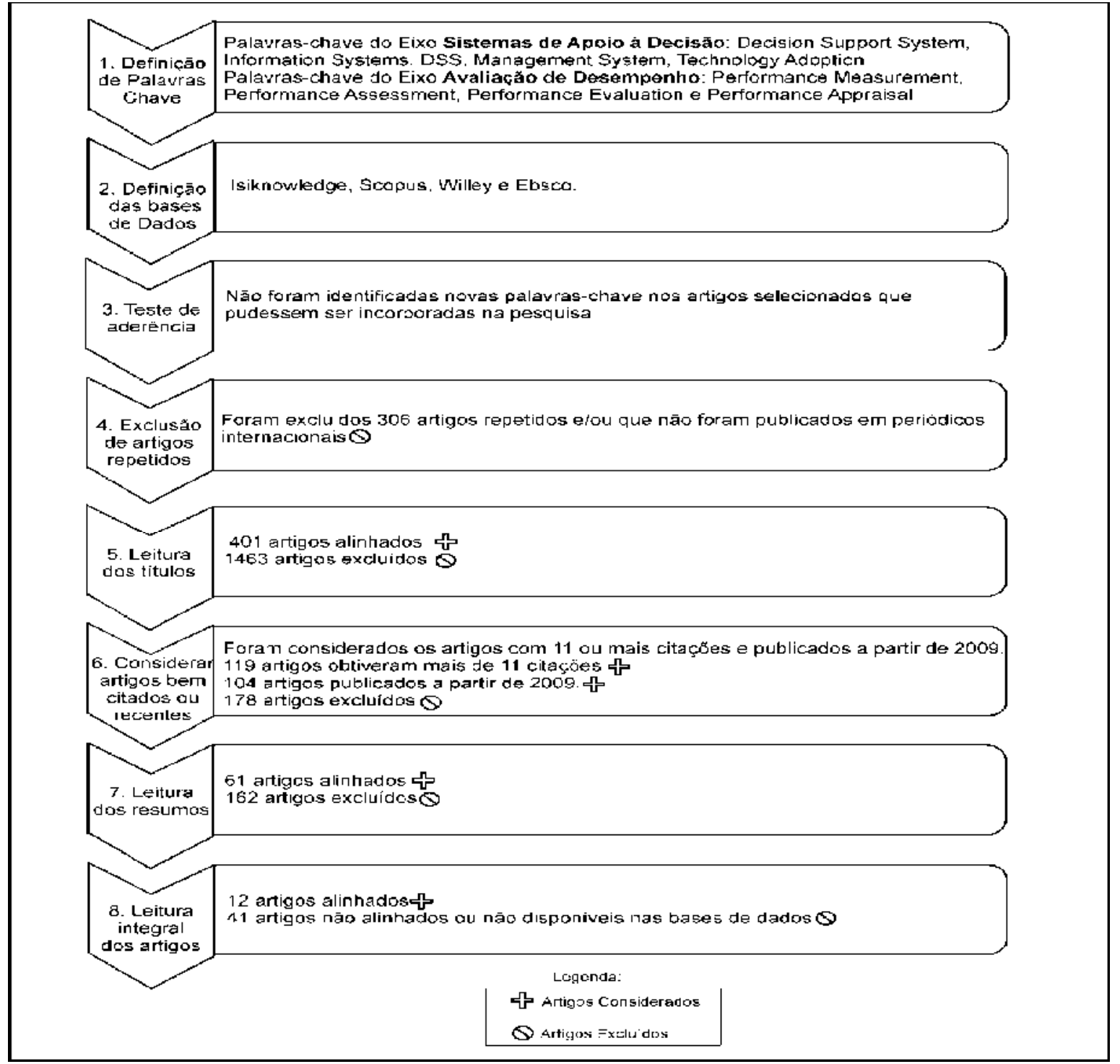

Fonte: Adaptado de Tasca et al.(2010).

Após a realização da consulta nas 4 bases de dados, obteve-se um resultado de 2.170 artigos. Desses artigos, foram escolhidos, a priori, 2 alinhados ao tema para realização do teste de aderência, a fim de verificar se apareciam novas palavras alinhadas ao tema e reiniciar a busca, incorporando essas palavras-chave. Após a leitura, constatou-se que nenhum dos artigos trazia novas palavras-chave alinhadas ao tema. Os 2.170 artigos fazem parte do banco de artigos. Conforme Bortoluzzi et al. (2011): “A primeira etapa da fase de filtragem do banco de artigos, refere-se à importação dos artigos para um software de gerenciamento bibliográfico".

Os 2.170 artigos, que representam os resultados da pesquisa, foram exportados para o software Endnote X3. Mesmo efetuando filtros referentes a artigos científicos, surgiram alguns de outras origens, que foram excluídos, assim como artigos duplicados. Restaram 1.864 artigos. 
Partiu-se, então, para a próxima etapa, a de exclusão de artigos com títulos não alinhados ao tema.

Entre os 1.864 artigos, 1.463 não obtiveram títulos suficientemente alinhados à pesquisa, conforme a percepção dos pesquisadores. Restaram 401 artigos potenciais com o título alinhado.

Os 401 artigos potenciais foram consultados no Google Acadêmico para se chegar ao conhecimento do número de citações de cada um. Foi delimitado pelos pesquisadores que os artigos com mais citações, que contivessem $70 \%$ do número total de citações de todos os 401 artigos, continuariam a ser classificados como artigos potenciais. Os demais seriam sujeitos a uma reanálise.

Para a pesquisa, 119 artigos satisfizeram a condição imposta pelos pesquisadores, representando $70 \%$ do total de citações dos 401 artigos. 0 artigo com mais citações entre os 119 apresentou 193 citações, e o com menos citações do grupo aprovado apresentou 11 citações.

Os 282 outros artigos entre os 401 que não obtiveram o número mínimo de 11 citações, foram sujeitos a uma reavaliação. Foram considerados, na pesquisa, artigos publicados a partir de 2009 como recentes, razão pela qual, entendeu-se, não tiveram tempo suficiente para atingir as 11 citações. Esses, então, foram reconsiderados para o processo. Foram aproveitados 104 artigos recentes entre os 282. Dessa forma, restaram 223 artigos potenciais, 104 recentes e 119 com representatividade em citações.

A próxima fase consistiu na leitura dos resumos dos artigos. Entre os 223, foram considerados 61 alinhados ao tema de pesquisa.

Para a etapa final, foi realizada a leitura integral dos artigos, sendo que, entre os 61 artigos, 53 estavam disponíveis, na íntegra, pelas bases de dados e foram sujeitos a uma leitura integral. Entre esses 53, 12 foram considerados alinhados à pesquisa. Esses artigos compõem o PB da pesquisa, conforme expõe o quadro 1.

Quadro 1 - Artigos do PB

1. CATERINO, N.; IERVOLINO, I.; MANFREDI, G.; COSENZA, E. Comparative analysis of multi-criteria decision-making methods for seismic structural retrofitting. Computer-Aided Civil and Infrastructure Engineering, v. 24, n. 6, p. 432-445, 2009.

2. CHALMETA, R.; GRANGEL, R. Performance measurement systems for virtual enterprise integration. International Journal of Computer Integrated Manufacturing, v. 18, n. 1, p. 73-84, Jan-Feb 2005. ISSN 0951-192X.

3. CHOY, K. L.; LEE, W. B.; LAU, H. C. W.; CHOY, L. C. A knowledge-based supplier intelligence retrieval 
system for outsource manufacturing. Knowledge-Based Systems, v. 18, n. 1, p. 1-17, Feb 2005. ISSN 0950-7051.

4. DEGRAEVE, Z.; ROODHOOFT, F.; VAN DOVEREN, B. The use of total cost of ownership for strategic procurement: A company-wide management information system. Journal of the Operational Research Society, v. 56, n. 1, p. 51-59, 2005.

5. EL HANANDEH, A.; EL-ZEIN, A. The development and application of multi-criteria decision-making tool with consideration of uncertainty: The selection of a management strategy for the bio-degradable fraction in the municipal solid waste. Bioresource Technology, v. 101, n. 2, p. 555-561, 2010.

6. GOMES, C. F. S.; NUNES, K. R. A.; XAVIER, L. H.; CARDOSO, R.; VALLE, R. Multicriteria decision making applied to waste recycling in Brazil. Omega-International Journal of Management Science, $\mathrm{v}$. 36, n. 3, p. 395-404, Jun 2008. ISSN 0305-0483.

7. HUNG, K. C.; JULIAN, P.; CHIEN, T.; JIN, W. T. H. A decision support system for engineering design based on an enhanced fuzzy MCDM approach. Expert Systems with Applications, v. 37, n. 1, p. 202213, Jan 2010. ISSN 0957-4174.

8. KENNERLEY, M.; NEELY, A. Enterprise resource planning: Analysing the impact. Integrated Manufacturing Systems, v. 12, n. 2, p. 103-113, 2002.

9. LEE, A. H. I.; CHEN, W. C.; CHANG, C. J. A fuzzy AHP and BSC approach for evaluating performance of IT department in the manufacturing industry in Taiwan. Expert Systems with Applications, v. 34, n. 1, p. 96-107, 2008.

10. WEN, W.; CHEN, Y. H.; CHEN, I. C. A knowledge-based decision support system for measuring enterprise performance. Knowledge-Based Systems, v. 21, n. 2, p. 148-163, 2008. ISSN 09507051.

11. XIROGIANNIS, G.; CHYTAS, P.; GLYKAS, M.; VALIRIS, G. Intelligent impact assessment of HRM to the shareholder value. Expert Systems with Applications, v. 35, n. 4, p. 2017-2031, Nov 2008. ISSN 09574174.

12. XU, D.-L.; YANG, J.-B. Intelligent decision system for self-assessment. Journal of Multi-Criteria Decision Analysis, v. 12, n. 1, p. 43-60, 2003. ISSN 1099-1360.

Fonte: Elaborado pelos autores, 2011.

\subsection{BIBLIOMETRIA}

Realizou-se a análise bibliométrica nos artigos do Portfólio Bibliográfico, apresentado

no

Quadro 1, para saber quais são os mais destacados periódicos, artigos e autores; bem como foi calculado o potencial do assunto, conforme o PB elaborado pelos pesquisadores em termos de fator de impacto.

Embora o processo de seleção de artigos tenha sido realizado entre março e abril de 2011, a bibliometria apresentada nesta seção foi atualizada em abril de 2012, para que a pesquisa se mantivesse com dados atualizados. 


\subsubsection{Periódicos de destaque}

Os periódicos do Portfólio Bibliográfico foram analisados em relação aos fatores de impacto definidos pelas bases Isiknowledge e Scopus, que são o Journal Citations Report (JCR) e Scopus Journal Report (SJR) respectivamente. Ressalta-se que, entre os 9 periódicos do PB, 7 possuem indexação nas 2 bases.

Figura 6-Periódicos com destaque em fator de impacto

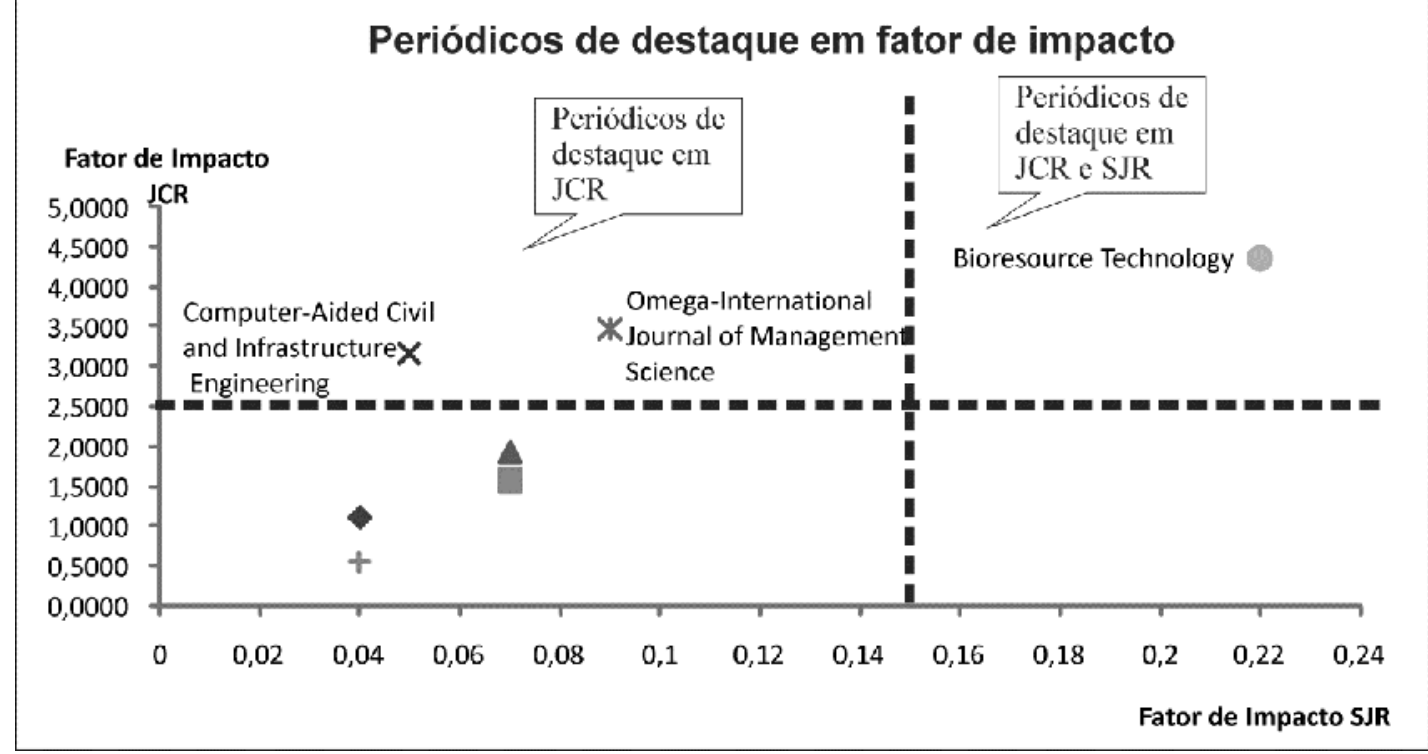

Fonte: Elaborado pelos autores, 2012.

O periódico Bioresource Technology, representado por um artigo que aborda a gestão da utilização de resíduos biodegradáveis na cidade de Sidney, destacou-se em fatores de impacto JCR e SJR, e tem um JCR de 4,3650 e um SJR de 0,22. Os periódicos Omega-International Journal of Management Science e Computer-Aided Civil and Infrastructure Engineering se destacaram em termos de JCR fatores de impacto de 3,467 e 3,17 respectivamente. Pode-se obsevar que, como foi mencionado na introdução deste trabalho, o processo decisório é multidisciplinar e, por isso, está presente em diferentes áreas, como se pode observar no escopo dos periódicos do PB.

\subsubsection{Artigos de destaque}


Nesta seção, os artigos do PB foram analisados conforme o número de citações dos artigos no Google acadêmico e o ano em que foram publicados. Posteriormente, serão analisados conforme o indicador citações/ano.

Figura 7 - Artigos de destaque em citações e tempo de publicação

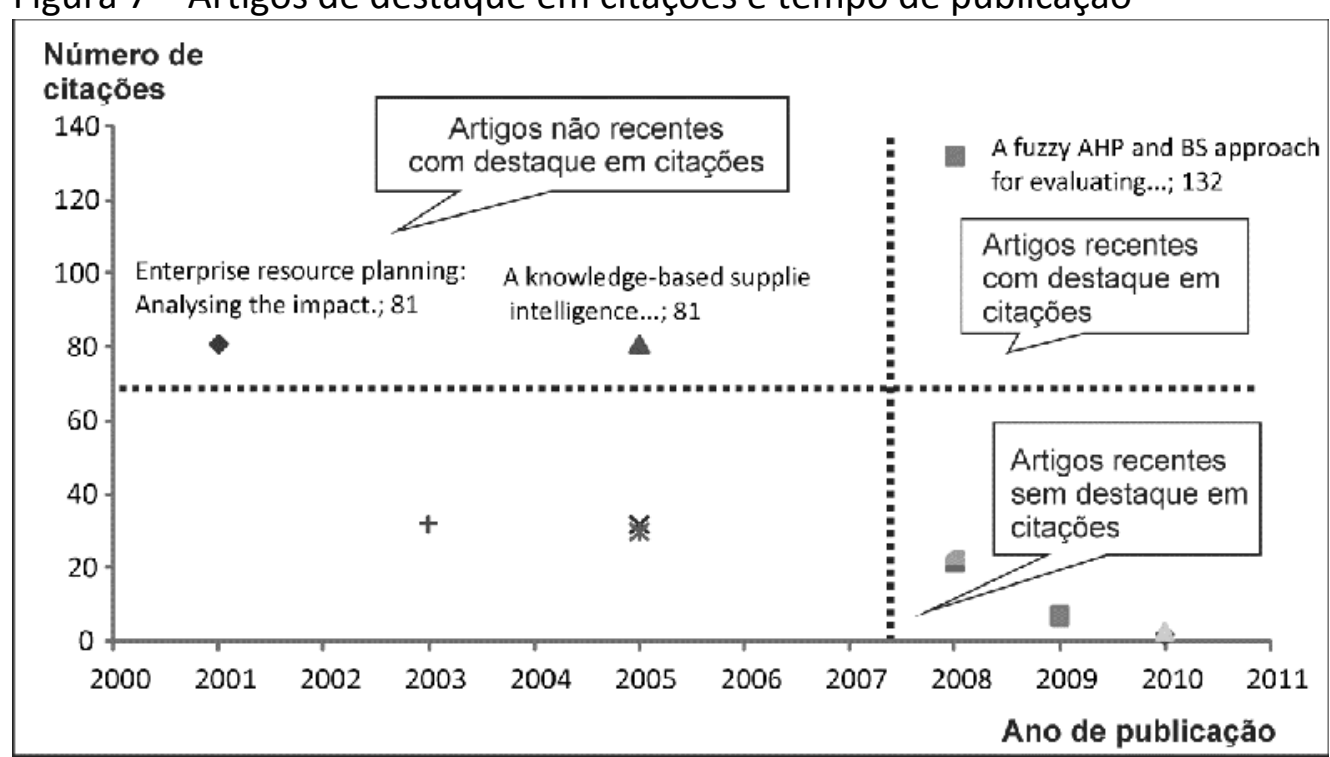

Fonte: Elaborado pelos autores, 2012.

O artigo A fuzzy AHP and BSC approach for evaluating performance of IT department in the manufacturing industry in Taiwan foi o mais citado, com 132 citações, tendo sido publicado em 2008. Em seguida, com 81 citações, aparecem os artigos Enterprise resource planning: Analysing the impact e $A$ knowledge-based supplier intelligence retrieval system for outsource manufacturing de 2001 e 2005 respectivamente. Essas análises podem ser vistas sob a ótica de citações por ano, como ilustra a Gráfico 1. O gráfico de citações por ano permite gerar entendimento sobre a média de citações por ano que o artigo tem. Para calcular esse índice, dividiu-se o número de citações que um artigo tem pela idade de publicação.

Gráfico 1 - Artigos de destaque em citações ano 


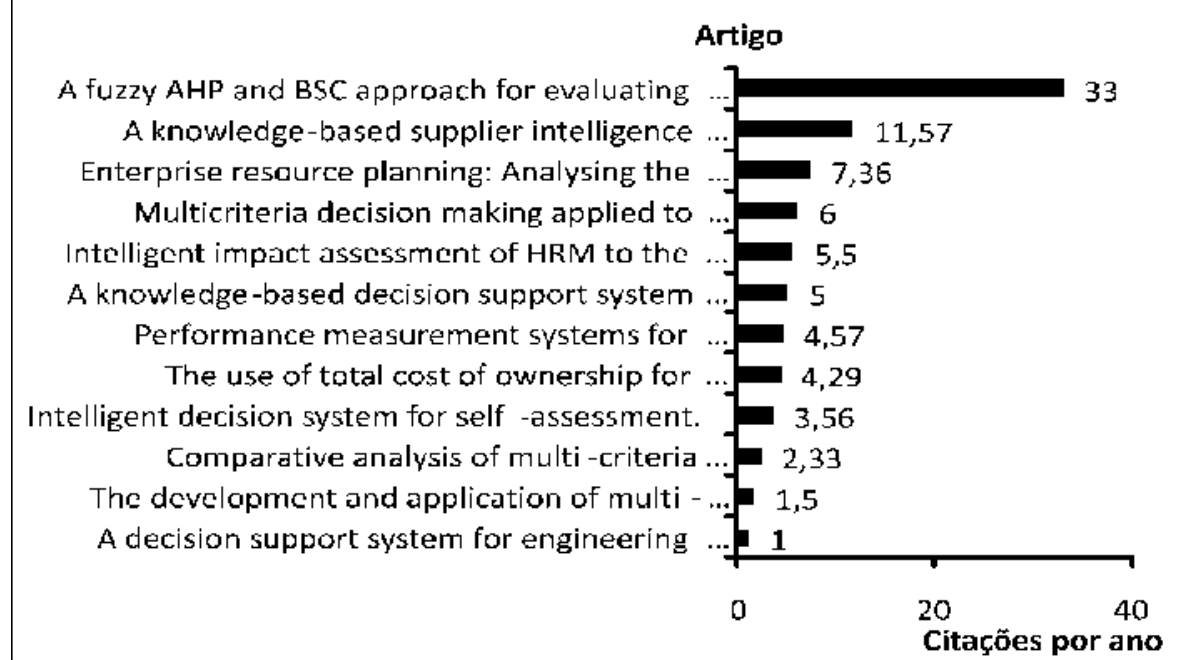

Fonte: Elaborado pelos autores, 2012.

Confrontando-se o gráfico 1 com o gráfico da Figura 7, os três artigos que mais têm número de citações são também os que têm mais número de citações/ano.

\subsubsection{Autores de destaque}

Entre os autores do Portfólio Bibliográfico, nenhum está presente em mais de um artigo, sendo assim, não há destaque. Desse modo, a análise de destaque dos autores está direcionada àqueles citados por diferentes artigos do PB.

Figura 8 - Relevância dos autores mais citados no PB

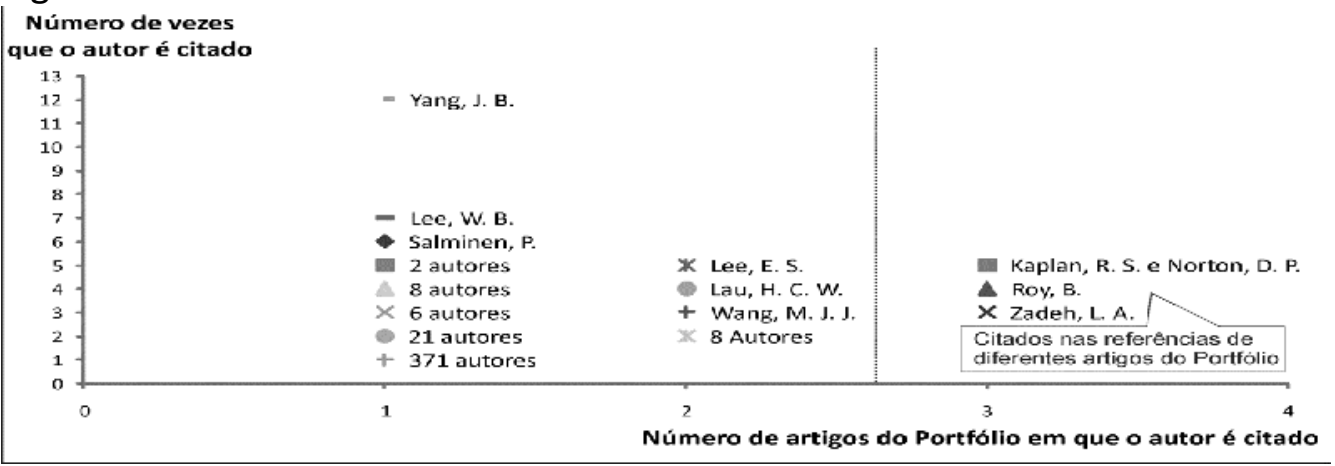

Fonte: Elaborado pelos autores, 2012.

Os autores Kaplan, R.S. e Norton, D.P, autores de The balanced scorecard-measures that drive performance, Roy, B., autor de ELECTRE III: Un algorithme de classement fondé sur une représentation floue des préférences en présence de critères multiples e Zadeh, L.A., autor de Fuzzy 
Sets apresentam 5, 4 e 3 citações respectivamente. Apesar de não serem os mais citados do PB, são os mais citados por diferentes artigos do PB e, por isso, são os autores de destaque.

\section{CONCLUSÕES}

Em face do processo decisório, sua multidisciplinaridade e importância no ambiente competitivo, emergiu a pergunta de pesquisa: "Como realizar um mapeamento sobre o tema Gestão do Processo Decisório conforme as delimitações postas pelos pesquisadores?", que estabeleceu o objetivo geral deste trabalho.

Assim, para atender o objetivo geral, foram delimitados os seguintes objetivos específicos:

(i) realizar um levantamento de artigos científicos publicados em periódicos internacionais a respeito do tema "Gestão do Processo decisório" para compor um Portfólio Bibliográfico, conforme as delimitações postas pelos pesquisadores;

(ii) efetuar uma bibliometria no PB para ressaltar os periódicos com maior fator de impacto, artigos mais citados e autores mais citados pelo PB.

O objetivo específico (i) foi cumprido, conforme relatado na seção 0 , quando foi evidenciado o processo ProKnow-C. O PB foi evidenciado no

Quadro 1.

A partir dos dados disponibilizados pelo PB, o objetivo específico (ii) foi atendido na seção 0. Através desse objetivo, foi possível saber quais são os principais periódicos, artigos e autores conforme as perspectivas dos pesquisadores, concluindo-se como destaques:

$\checkmark$ Periódico com Maior Journal Report Citations (JCR): Bioresource Technology com JCR igual a 4,36 .

$\checkmark$ Periódico com Maior SCImago Journal Rank (SJR): Bioresource Technology com SJR de 0,22 .

Artigo com destaque em citações: Lee et al. (2008), com 132 citações e uma média de 33 citações por ano.

$\checkmark$ Autores de destaque: Kaplan, R.S e Norton, D.P., Roy, B. e Zadeh, L.A.,que foram citados por 3 diferentes artigos do PB. 
Dessa maneira, para realizar o mapeamento do tema Gestão do Processo Decisório, foi identificado um PB, conforme as delimitações postas pelos pesquisadores, desde as palavraschave a serem utilizadas como filtros sobre alinhamento dos títulos, resumos e alinhamento completo do artigo. Posteriormente, procedeu-se com um levantamento dos principais periódicos e artigos do PB e os autores mais citados no PB.

Assim como as pesquisas de Bortoluzzi et al. (2011) e Junior et al. (2012), foi possível compreender melhor o assunto por meio de um PB definido pelas delimitações postas pelos pesquisadores e, através do PB e respectivas referências, foi possível um melhor entendimento sobre publicações referentes ao assunto.

Ressaltada a importância da gestão do processo decisório e sua presença em diferentes áreas do conhecimento, esta pesquisa contribui com a elaboração de um Portfólio Bibliográfico referente ao tema, possibilitando a geração de conhecimento sobre variáveis relacionadas, o que orientará futuros trabalhos.

Para pesquisas futuras, recomenda-se a realização de uma análise de conteúdo do PB, baseada numa visão de mundo em que seja exposta a origem dos critérios a serem tidos em conta.

O trabalho apresentou como delimitação uma pesquisa realizada nos portais disponíveis pela CAPES, recomendando-se, também, para futuros trabalhos, uma análise de conteúdo sobre o PB, baseada em uma afiliação teórica.

\section{DECISIONAL PROCESS MANAGEMENT: KNOWLEDGE CONSTRUCTION ABOUT THE FIELD}

\section{ABSTRACT}

The decision support management has a large scope and that's why it has called the researchers attention. Therefore, this theme worth an investigation. The aim of this paper, fit as exploratorydescriptive, is to perform a mapping under the decisional process management according to the delimitations establish by the researchers. To accomplish the purpose will be used Knowledge Development Process - Constructivist (ProKnow-C) as intervention tool. The process enabled us to identify a Bibliographic Portfolio (BP) composed of 12 articles aligned to the researchers' perceptions and delimitations. Through this BP and its references we could disclosure the 
outstanding: (i) Journals with highest impact factor; (ii) The most cited articles; (iii) The most cited authors and; (iv) the BP distribution related to Journal Report Citattions - JCR and SCImago Journal Rank - SJR impact factors.

Key-words: Decision Support. Performance Evaluation. Bibliometric Analysis. Management.

\section{REFERÊNCIAS}

AFONSO, M.H.F.; SOUZA, J.V.; ENSSLIN, S.R.; ENSSLIN, L. Como construir conhecimento sobre o tema de pesquisa? Aplicação do processo proknow-c na busca de literatura sobre avaliação do desenvolvimento sustentável. Revista de Gestão Social e Ambiental, v. 5, n. 2, 2011. ISSN 1981$982 \mathrm{X}$.

BOOTH, A.; CLARKE, M.; GHERSI, D.; MOHER, D.; PETTICREW, M. e STEWART, L. Establishing a Minimum Dataset for Prospective Registration of Systematic Reviews: An International Consultation. PLoS ONE, v. 6, n. 11, 2011. ISSN 1932-6203.

BORTOLUZZI, S.C.; ENSSLIN, S.R.; ENSSLIN, L.; VALMORBIDA, S.M.I. Avaliação de Desempenho em Redes de Pequenas e Médias Empresas: Estado da arte para as delimitações postas pelo pesquisador. Revista Eletrônica de Estratégia \& Negócios, v. 4, n. 2, p. 202-222, 2011. ISSN 19843372.

BRANDT, R.; WARD, C. L.; DAWES, A. e FLISHER, A. J. Epidemiological measurement of children's and adolescents' exposure to community violence: Working with the current state of the science. Clinical Child and Family Psychology Review, v. 8, n. 4, p. 327-342, Dec 2005. ISSN 1096-4037.

CALLCUT, R. A.; BRANSON, R. D. How to Read a Review Paper. Respiratory Care, v. 54, n. 10, p. 1379-1385, 2009. ISSN 0020-1324.

CATERINO, N.; IERVOLINO, I.; MANFREDI, G.; COSENZA, E. Comparative analysis of multi-criteria decision-making methods for seismic structural retrofitting. Computer-Aided Civil and Infrastructure Engineering, v. 24, n. 6, p. 432-445, 2009.

CHALMETA, R.; GRANGEL, R. Performance measurement systems for virtual enterprise integration. International Journal of Computer Integrated Manufacturing, v. 18, n. 1, p. 73-84, Jan-Feb 2005. ISSN 0951-192X.

CHOY, K. L.; LEE, W. B.; LAU, H. C. W.; CHOY, L. C. A knowledge-based supplier intelligence retrieval system for outsource manufacturing. Knowledge-Based Systems, v. 18, n. 1, p. 1-17, Feb 2005. ISSN 0950-7051. 
DA ROSA, F.S.; ENSSLIN, S.R.; ENSSLIN, L.; LUNKES, R.J. Gestão da evidenciação ambiental: um estudo sobre as potencialidades e oportunidades do tema. Engenharia Sanitária Ambiental, v. 16, n. 1, p. 157-166, 2011.

DE MORAES, L.; GARCIA, R.; ENSSLIN, L.; DA CONCEIÇÃO, M.J.; DE CARVALHO, S.M. The multicriteria analysis for construction of benchmarkers to support the Clinical Engineering in the Healthcare Technology Management. European Journal of Operational Research, v. 200, n. 2, p. 607-615, 2010. ISSN 0377-2217.

DEGRAEVE, Z.; ROODHOOFT, F.; VAN DOVEREN, B. The use of total cost of ownership for strategic procurement: A company-wide management information system. Journal of the Operational Research Society, v. 56, n. 1, p. 51-59, 2005.

EL HANANDEH, A.; EL-ZEIN, A. The development and application of multi-criteria decision-making tool with consideration of uncertainty: The selection of a management strategy for the biodegradable fraction in the municipal solid waste. Bioresource Technology, v. 101, n. 2, p. 555-561, 2010.

ESSLIN, L.; ENSSLIN, S. R.; LACERDA, R. T. O.; TASCA, J. E. ProKnow-C, Knowledge Development Process-Constructivist. Processo técnico com patente de registro pendente junto ao INPI. Brasil, 2010a.

ENSSLIN, L.; ENSSLIN, S. R. e PACHECO, G. C. Um estudo sobre segurança em estádios de futebol baseado na análise bibliométrica da literatura internacional. Perspectivas em Ciência da Informação, v. 17, n. 2, p. 71-91, 2012. ISSN 1981-5344.

ENSSLIN, L.; GIFFHORN, E.; ENSSLIN, S. R.; PETRI, S. M.; VIANNA, W. B. Avaliação do Desempenho de Empresas Terceirizadas com o Uso da Metodologia Multicritério de Apoio à Decisão-

Construtivista. Revista Pesquisa Operacional, v. 30, n. 1, p. 125-152, Janeiro a Abril de 2010b. Versão impressa ISSN 0101-7438 / versão online ISSN 1678-5142.

FERREIRA, N. S.; YOSHIDA, E. M. P. Produção científica sobre psicoterapias breves no Brasil e demais países latinoamericanos (1990-2000). Est. Psicol, v. 3, n. 3, p. 523-531, 2004.

GOMES, C. F. S.; NUNES, K. R. A.; XAVIER, L. H.; CARDOSO, R.; VALLE, R. Multicriteria decision making applied to waste recycling in Brazil. Omega-International Journal of Management Science, v. 36, n. 3, p. 395-404, Jun 2008. ISSN 0305-0483.

GUNASEKARAN, A.; PATEL, C.; MCGAUGHEY, R. E. A framework for supply chain performance measurement. International Journal of Production Economics, v. 87, n. 3, p. 333-347, 2004. ISSN 0925-5273.

HIRST, W. M.; LE FEVRE, A. M.; LOGUE, D. N.; OFFER, J. E.; CHAPLIN, S. J.; MURRAY, ; D.; WARD, W. R. e FRENCH, N. P. A systematic compilation and classification of the literature on lameness in cattle. Veterinary Journal, v. 164, n. 1, p. 7-19, 2002. ISSN 1090-0233. 
HOFFMAN, J. J.; COUCH, G.; LAMONT, B. T. The effect of firm profit versus personal economic well being on the level of ethical responses given by managers. Journal of Business Ethics, v. 17, n. 3, p. 239-244, 1998.

HUNG, K. C.; JULIAN, P.; CHIEN, T.; JIN, W. T. H. A decision support system for engineering design based on an enhanced fuzzy MCDM approach. Expert Systems with Applications, v. 37, n. 1, p. 202-213, 2010.

IMBERGER, G.; VEJLBY, A. D. ; HANSEN, S. B.; MOLLER, A. M. e WETTERSLEV, J. Statistical Multiplicity in Systematic Reviews of Anaesthesia Interventions: A Quantification and Comparison between Cochrane and Non-Cochrane Reviews. PLoS ONE, v. 6, n. 12, 2011.

IUDÍCIBUS, S. Teoria da Contabilidade. São Paulo: 2004.

JUNIOR, E. D. B.; ENSSLIN, L.; ENSSLIN, S. R. Seleção e análise de um portfólio de artigos sobre avaliação de desempenho na cadeia de suprimentos. GEPROS - Gestão da Produção, n. 1, 2012

KATSIKOPOULOS, K. V. Psychological Heuristics for Making Inferences: Definition, Performance, and the Emerging Theory and Practice. Decision Analysis, v. 8, n. 1, p. 10-29, 2011. ISSN 15458490 .

KENNERLEY, M.; NEELY, A. Enterprise resource planning: Analysing the impact. Integrated Manufacturing Systems, v. 12, n. 2, p. 103-113, 2002.

KNOBLOCH, K.; YOON, U.; VOGT, P. M. Preferred reporting items for systematic reviews and metaanalyses (PRISMA) statement and publication bias. Journal of Cranio-Maxillofacial Surgery, v. 39, n. 2, p. 91-92, 2011. ISSN 1010-5182.

LACERDA, R. T. O.; ENSSLIN, L.; ENSSLIN, S. R. Contribuições à gestão estratégica de organizações quando analisados na visão de seu desempenho. GESTÃo. Org-Revista Eletrônica de Gestão Organizacional, v. 2, n. 9, 2011. ISSN 1679-1827.

LAKATOS, E. M.; MARCONI, M. Fundamentos de metodologia científica. São Paulo: Atlas, 2008. ISBN 8522440158.

LEE, A. H. I.; CHEN, W. C.; CHANG, C. J. A fuzzy AHP and BSC approach for evaluating performance of IT department in the manufacturing industry in Taiwan. Expert Systems with Applications, v. 34, n. 1, p. 96-107, 2008.

LIBERATI, A.; ALTMAN, D. G.; TETZLAFF, J.; MULROW, C.; GOTZSCHE, P. C.; IOANNIDIS, J. P. A.; CLARKE, M.; DEVEREAUX, P. J.; KLEIJNEN, J.; MOHER, D. The PRISMA Statement for Reporting Systematic Reviews and Meta-Analyses of Studies That Evaluate Health Care Interventions: Explanation and Elaboration. Plos Medicine, v. 6, n. 7, Jul 2009.

LITTELL, J. H. Evidence-based or biased? The quality of published reviews of evidence-based practices. Children and Youth Services Review, v. 30, n. 11, p. 1299-1317, 2008. ISSN 0190-7409. 
LOKEN, E. Use of multicriteria decision analysis methods for energy planning problems. Renewable \& Sustainable Energy Reviews, v. 11, n. 7, p. 1584-1595, 2007.

LUNKES, R. J.; VICENTE, M. R. F.; DA ROSA, F. S. Pesquisa científica em contabilidade gerencial: Estudo comparativo entre Espanha e Brasil. Revista Contaduría y Administración, v. 57, n. 2, p. 159-184, 2012.

MACLURE, M. 'Clarity bordering on stupidity': where's the quality in systematic review? Journal of Education Policy, v. 20, n. 4, p. 393-416, 2005. ISSN 0268-0939.

MARCONDES, C.H.; MENDONÇA, M.A.R.; MALHEIROS, L.R.; COSTA, L.C.; SANTOS, T.C.P. Ontologies as the new bases of scientific knowledge. Perspectivas em Ciência da Informação, v. 13, n. 3, p. 20-39, 2008. ISSN 1413-9936.

MARTIN, N. C. Da contabilidade à controladoria: a evolução necessária. Revista Contabilidade \&amp; Finanças, v. 13, n. 28, p. 7-28, 2002.

MORGAN, S.; GROOTENDORST, P.; LEXCHIN, J.; CUNNINGHAM, C. e GREYSON, D. The cost of drug development: A systematic review. Health Policy, v. 100, n. 1, p. 4-17, 2011. ISSN 0168-8510.

ORDOOBADI, S. M. Development of a supplier selection model using fuzzy logic. Supply Chain Management-an International Journal, v. 14, n. 4, p. 314-327, 2009. ISSN 1359-8546.

PATEL, R. V.; SINUFF, T.; COOK, D. J. Influencing advance directive completion rates in nonterminally ill patients: A systematic review. Journal of Critical Care, v. 19, n. 1, p. 1-9, 2004. ISSN 0883-9441.

SALAS, E.; ROSEN, M. A.; DIAZGRANADOS, D. Expertise-Based Intuition and Decision Making in Organizations. Journal of Management, v. 36, n. 4, p. 941-973, 2010.

RICHARDSON, R. J. Pesquisa social, métodos e técnicas. São Paulo: Atlas, 1999.

. Pesquisa social, métodos e técnicas. 3. ed. São Paulo: Atlas, 2008.

ROJON, C.; MCDOWALL, A.; SAUNDERS, M. N. K. On the Experience of Conducting a Systematic Review in Industrial, Work, and Organizational Psychology Yes, It Is Worthwhile. Journal of Personnel Psychology, v. 10, n. 3, p. 133-138, 2011. ISSN 1866-5888.

ROY, B. On operational research and decision aid. European Journal of Operational Research, v. 73, n. 1, p. 23-26, 1994.

STERNBERG, R. J. Successful intelligence as a basis for entrepreneurship. Journal of Business Venturing, v. 19, n. 2, p. 189-201, 2004.

STRECH, D.; PERSAD, G.; MARCKMANN, G. E DANIS, M. Are physicians willing to ration health care? Conflicting findings in a systematic review of survey research. Health Policy, v. 90, n. 2-3, p. 113124, 2009. ISSN 0168-8510. 
TASCA, J.E.; ENSSLIN, L.; ENSSLIN, S.R.; ALVES, M.B.M. An approach for selecting a theoretical framework for the evaluation of training programs. Journal of European Industrial Training, v. 34, n. 7, p. 631-655, 2010. ISSN 0309-0590.

THOMPSON, R. L.; BANDERA, E. V.; BURLEY, V. J.; CADE, J. E.; FORMAN, D.; FREUDENHEIM, J. L.; GREENWOOD, D.; JACOBS, D. R.; KALLIECHARAN, R. V.; KUSHI, L. H.; MCCULLOUGH, M. L.; MILES, L. M.; MOORE, D. F.; MORETON, J. A.; RASTOGI, T.; WISEMAN, M. J. Reproducibility of systematic literature reviews on food, nutrition, physical activity and endometrial cancer. Public Health Nutrition, v. 11, n. 10, p. 1006-1014, Oct 2008.

TRICCO, A. C.; STRAUS, S. E.; MOHER, D. How can we improve the interpretation of systematic reviews? Bmc Medicine, v. 9, 2011. ISSN 1741-7015.

TRICCO, A. C.; STRAUS, S. E.; MOHER, D. How can we improve the interpretation of systematic reviews? Bmc Medicine, v. 9, 2011. ISSN 1741-7015.

VALMORBIDA, S.M.I.; ENSSLIN, S. R.; ENSSLIN, L; BORTOLUZZI, S.C. Gestão Pública com foco em resultados: Evidenciação de oportunidades de pesquisa. CAP-Accounting and Management, v. 5, n. 5, p. 126-136, 2011.

VIANNA, W. B.; ENSSLIN, L.; GIFFHORN, E. A integração sistêmica entre pós-graduação e educação básica no Brasil: Contribuição teórica para um "estado da arte". Ensaio: Avaliação e Políticas Públicas em Educação, v. 19, n. 71, p. 327-344, 2011. ISSN 0104-4036.

VILAN FILHO, J. L.; SOUZA, H. B.; MUELLER, S. Articles from Brazilian scientific journals in information areas: evolution of production and multiple authorship. Perspectivas em Ciência da Informação, v. 13, n. 2, p. 2-17, 2008. ISSN 1413-9936.

WEN, W.; CHEN, Y. H.; CHEN, I. C. A knowledge-based decision support system for measuring enterprise performance. Knowledge-Based Systems, v. 21, n. 2, p. 148-163, 2008. ISSN 09507051.

XIROGIANNIS, G.; CHYTAS, P.; GLYKAS, M.; VALIRIS, G. Intelligent impact assessment of HRM to the shareholder value. Expert Systems with Applications, v. 35, n. 4, p. 2017-2031, Nov 2008. ISSN 0957-4174.

XU, D.-L.; YANG, J.-B. Intelligent decision system for self-assessment. Journal of Multi-Criteria Decision Analysis, v. 12, n. 1, p. 43-60, 2003. ISSN 1099-1360. 